DOI: https://doi.org/10.17162/rccs.v11i2.1107

\title{
Empatía y Creencias Irracionales en Internos por delito contra la libertad sexual de un instituto penitenciario de Lima Este
}

\section{Empathy and irrational beliefs imprisoned for crimes against the sexual freedom of a penitentiary institute in Lima East.}

\author{
Lisseth Casariego More', Rudy Jara Cueto²
}

\begin{abstract}
RESUMEN
Objetivo analizar la relación entre las variables empatía y creencias irracionales, Materiales y métodos: es de diseño no experimental, de corte transversal y alcance correlacional. La muestra estuvo compuesta por 127 internos en un instituto penitenciario de Lima Este, mayores de 18 años, recluidos por delito contra la libertad sexual, Los instrumentos utilizados para la recolección de datos fueron la Escala de Reactividad Interpersonal (Davis, 1980) y el Registro de Opiniones (Davis, McKay y Eshelman, 1987). Resultados evidenciaron que sí existe relación significativa inversa entre empatía y las creencias irracionales de competencia absoluta y continuidad de efectos $(p<0.05)$. Sin embargo, no se encontró relación significativa entre empatía y las creencias irracionales de afecto y aprobación, relatividad de los actos, expectativas sociales y control externo $(p>0.05)$. Conclusiones: la empatía está vinculada de manera inversa a la autosuficiencia generada por la creencia de ser absolutamente competente y el inadecuado afrontamiento de experiencias del pasado.
\end{abstract}

Palabras claves: Empatía, creencias irracionales, delito, contra la libertad sexual.

\section{ABSTRACT}

Objective of the present investigation was to analyze the relationship between the variables of empathy and irrational beliefs. Materials and methods: it is of non-experimental design, of crosssection and correlational scope. The sample consisted of 127 inmates in a penitentiary institute in Lima East, over 18 years of age, held for crimes against sexual freedom. The instruments used for data collection were the Interpersonal Reactivity Scale (Davis, 1980) and the Registry of Opinions (Davis, McKay and Eshelman, 1987). Results showed that there is a significant inverse relationship between empathy and the irrational beliefs of absolute competence and continuity of effects $(p<0.05)$. However, no significant relationship was found between empathy and the irrational beliefs of affection and approval, relativity of acts, social expectations and external control ( $p>0.05)$. Conclussion: that empathy is inversely linked to self-sufficiency generated by the belief of being absolutely competent and the inadequate coping of past experiences.

Keywords: Empathy, irrational beliefs, crime, against sexual freedom.

${ }^{1}$ Psicóloga, Consultorio Psicológico particular, Lima, Perú.

${ }^{2}$ Psicólogo, Posta Médica de Tocache, San Martín, Perú. 


\section{INTRODUCCIÓN}

Actualmente es habitual escuchar en los medios de comunicación noticias relacionadas a los delitos contra la libertad sexual. Al respecto la Organización de las Naciones Unidas (ONU, 2015) y la Organización Mundial de la Salud (OMS, 2015) refieren que 1 de cada 10 niñas en el mundo han sido víctimas de abuso sexual, en relación a Perú en los en los últimos años se ha incrementado notablemente la violencia hacia la mujer. En este sentido el $37 \%$ de las mujeres entre 15 y 49 años mencionan que alguna vez han sido víctima de violencia física o sexual (Instituto Nacional de Estadística e Informática [INEI], 2015). Además, se menciona que el $90 \%$ de las víctimas son menores de edad, las cuales han sido agredidas o forzadas por personas cercanas a su entorno social (Ministerio de la Mujer y Poblaciones Vulnerables [MIMP], 2015).

Esta situación ha generado en los investigadores la incógnita del porqué la frecuencia de esta situación. Partiendo de un análisis psicológico, el interés de la presente investigación se centra en el hecho de cómo la empatía y las creencias irracionales intervienen para que se dé este tipo de conductas delictivas.

Davis (1996) refiere que la empatía es la capacidad intelectual y emocional/afectiva para ponerse en el lugar del otro, tomando en cuenta la perspectiva de otra persona, así como las reacciones emocionales que se suscitan en medio de una situación específica. De igual forma menciona que está compuesta por dos principales procesos: cognitivos y emocionales. Dentro de los procesos cognitivos se encuentran las dimensiones toma de perspectiva y fantasía; y dentro de los procesos emocionales se encuentran las dimensiones preocupación empática y malestar personal.

Ellis y Harper (1975) mencionan que las creencias irracionales son errores en el procesamiento de la información y que se caracterizan por la presencia de distorsiones en la percepción de la realidad; esto debido a opiniones subjetivas, mas no por el valor de los hechos objetivos. Este tipo de situaciones ponen al bienestar y la seguridad personal en peligro, ya que impiden la obtención de metas claras; existe una tendencia a la exageración del malestar producido por una determinada situación y las relaciones interpersonales mayormente son inestables por la presencia de constantes conflictos.
En este sentido Tagle y Juárez (2014) consideran que los agresores sexuales se caracterizan por diversos factores significativos que llevan a cometer el delito, como la inadecuada empatía, las distorsiones cognitivas, la falta de control de impulsos y el uso de mecanismos para justificar acciones. En este sentido Ortiz, Sánchez y Cardenal (2002) mencionan que además de estas características, la inestabilidad emocional, la impulsividad y la dificultad para prever las consecuencias de sus actos conllevan a que los agresores sexuales posean un inadecuado pronóstico de adaptación, asimismo presentan rasgos de personalidad dependiente, antisocial y compulsiva.

En cuanto a la relación de ambas variables, Marshall (2004) menciona que los delincuentes sexuales presentan menores niveles de empatía que otras personas y que esta característica también la presenta frente a los abusos sexuales de otros delincuentes. Por otro lado, Fernández, Marshall, Lightbody y Sullivan (1999) mencionan que esta falta de empatía hacia la víctima se debe a la distorsión cognitiva de la situación, puesto que el agresor busca protegerse de los juicios negativos de sí mismo y de los demás.

En relación a antecedentes de esta investigación Cepeda (2012) realizó un estudio con el objetivo de analizar, explorar, describir y comparar los factores de empatía y distorsiones cognitivas en tres grupos: agresores sexuales de menores de edad, delincuentes violentos no sexuales y personas sin historia de reclusión en establecimientos carcelarios. Los resultados que se obtuvieron indicaron diferencias significativas en el factor afectivo y cognitivo de la empatía entre agresores sexuales y delincuentes violentos; los agresores sexuales reportaron menor empatía cuando la situación evalúa a sus propias víctimas y el grupo de comparación presentó mayor presencia de distorsiones cognitivas que los grupos de personas recluidas en el establecimiento carcelario.

Existe poca información sobre ambas variables y la población de estudio, por lo que se insta a ampliar el número de investigaciones de esta índole. Por todo lo anterior, el objetivo principal de esta investigación consistió en determinar si existe relación significativa entre empatía y creencias irracionales en internos por delito contra la libertad sexual de un instituto penitenciario de Lima Este. 


\section{MATERIALES Y MÉTODOS}

El diseño de la investigación fue realizada a través de un enfoque cuantitativo, diseño no experimental puesto que no se manipularon las variables y se recogió la información tal como se presentó. Así mismo, es de corte transversal, debido a que se recolectaron los datos en un solo momento; y es de alcance correlacional, debido a que se busca encontrar la relación de la empatía y las creencias irracionales en la población de estudio (Hernández, Fernández \& Baptista, 2006).

\section{Participantes:}

La muestra considerada para la investigación está compuesta por 127 internos del pabellón 16 del Instituto Penitenciario Lurigancho, de entre 20 a 65 años de edad, que se caracterizan por ser internos primarios (1er ingreso a un centro penitenciario), procesados y sentenciados por delitos contra la libertad sexual (violación sexual, actos contra el pudor y tentativa de violación); su grado de instrucción varía, teniendo en su mayoría estudios primarios y secundarios incompletos. En esta investigación se realizó un muestreo no probabilístico por conveniencia, en función a los sujetos a investigar, es decir se tuvo que realizar una cuidadosa y controlada elección de los sujetos con las características determinadas anteriormente (Hernández, Fernández \& Baptista, 2006).

En la tabla 1, se observa que el $67.7 \%$ de la población presentó edades que oscilaron entre 20 y 40 años de edad, mientras que el $32.3 \%$ tuvo de 41 a 65 años de edad. Asimismo, el $47.2 \%$ solteros y sólo el $13.4 \%$ estaban casados. Respecto al tipo de delito, la mayoría de los participantes (62.2\%) cometió agresión sexual en la modalidad de actos contra el pudor. En cuanto a la edad de la víctima de los agresores sexuales, se observa que la mayoría $(63 \%)$ estuvo en el rango de edad de 8 a 14 años de edad.

Tabla 1

Datos sociodemográficos de los participantes en el estudio.

\begin{tabular}{|c|c|c|c|}
\hline Variable & Categorías & Frecuencia & Porcentaje \\
\hline \multirow[t]{2}{*}{ Edad } & 20 a 40 años & 86 & $67.7 \%$ \\
\hline & 41 a 65 años & 41 & $32.3 \%$ \\
\hline \multirow[t]{3}{*}{ Grado de instrucción } & Primaria & 21 & $16.5 \%$ \\
\hline & Secundaria & 81 & $63.8 \%$ \\
\hline & Superior & 25 & $19.7 \%$ \\
\hline \multirow[t]{4}{*}{ Estado civil } & Soltero & 60 & $47.2 \%$ \\
\hline & Conviviente & 45 & $35.4 \%$ \\
\hline & Casado & 17 & $13.4 \%$ \\
\hline & Otro & 5 & $3.9 \%$ \\
\hline \multirow[t]{3}{*}{ Procedencia } & Costa & 73 & $57.5 \%$ \\
\hline & Sierra & 35 & $27.6 \%$ \\
\hline & Selva & 19 & $15 \%$ \\
\hline \multirow[t]{3}{*}{ Delito } & Violación sexual & 44 & $34.6 \%$ \\
\hline & Actos contra el pudor & 79 & $62.2 \%$ \\
\hline & Tentativa de violación & 4 & $3.1 \%$ \\
\hline \multirow[t]{4}{*}{ Edad de la victima } & 0 a 7 años & 20 & $15.7 \%$ \\
\hline & 8 a 14 años & 80 & $63 \%$ \\
\hline & 15 a 17 años & 16 & $12.6 \%$ \\
\hline & 18 a más & 11 & $8.7 \%$ \\
\hline \multirow[t]{3}{*}{ Tiempo de reclusión } & 0 meses a 1 año & 47 & $37 \%$ \\
\hline & 2 años a 7 años & 40 & $31.5 \%$ \\
\hline & 8 años a 13 años & 30 & $23.6 \%$ \\
\hline
\end{tabular}




\begin{tabular}{llcc}
\hline \multirow{2}{*}{ Situación jurídica } & 14 años a más & 10 & $7.9 \%$ \\
\cline { 2 - 3 } Tiempo de sentencia & Procesado & 37 & $29.1 \%$ \\
\cline { 2 - 3 } & Nentenciado hay sentencia & 90 & $70.9 \%$ \\
& 1 año a 6 años & 37 & $29.1 \%$ \\
& 7 años a 12 años & 26 & $20.5 \%$ \\
& 13 años a 20 años & 31 & $24.4 \%$ \\
& 21 años a 35 años & 19 & $15 \%$ \\
\hline
\end{tabular}

\section{Instrumentos:}

Los instrumentos utilizados fueron el Índice de Reactividad Interpersonal (IRI) propuesto por Davis (1980) y adaptada al español por Albéniz, Paúl, Etxeberría, Montes y Torres (2003); este instrumento se elaboró con la finalidad de medir la empatía disposicional en adultos. Está compuesto por 28 ítems los cuales son distribuidos de forma equitativa en cuatro subescalas que miden cuatro dimensiones del concepto global de empatía: dimensión toma de perspectiva, dimensión fantasía, la dimensión preocupación empática y malestar personal. Asimismo, menciona que las opciones de respuestas de la prueba se encuentran en una escala tipo Likert. Finalmente, con respecto a las propiedades psicométricas, en el presente estudio se realizó el análisis de fiabilidad para la muestra de estudio a través del coeficiente Alpha de Cronbach, y se obtuvo un coeficiente de, 508 .

Para medir las creencias irracionales se empleó, el Registro de Opiniones (REGOPINA) de Davis, McKay y Eshelman (1987) y adaptado en Perú por Guzman (1987). Este instrumento está compuesto por 65 ítems distribuidos de forma equitativa en los 6 indicadores de las creencias irracionales: creencia de afecto y aprobación, creencia de competencia absoluta, creencia de relatividad, creencia de expectativas sociales, creencia de control externo, creencia de continuidad de efectos y adicionalmente 5 ítems de consistencia, Así mismo, la opción de respuesta para la administración de este instrumento es tipo dicotómica ( $\mathrm{V} \circ \mathrm{F}$ ).

Finalmente, con respecto a las propiedades psicométricas, en el presente estudio se realizó el análisis de fiabilidad para la muestra de estudio a través del coeficiente Alpha de Cronbach, y se obtuvo un coeficiente de ,694.

\section{RESULTADOS}

En cuanto al análisis de correlación entre las variables de investigación. Se puede apreciar en la tabla 2 que el coeficiente de correlación de Spearman $p(r h o)$ indica que existe relación significativa entre el nivel de empatía y la creencia irracional "Debemos ser absolutamente competentes, inteligentes y eficaces en todos los aspectos posibles" $(r=-, 187, p<0.05)$, además se aprecia que existe una relación alta y negativa, es decir, cuanto mayor sea el grado de esta creencia, menor será el grado de empatía en los internos. De manera similar ocurre con la creencia "Si algo afectó fuertemente en alguna ocasión nuestra vida, siempre la seguirá afectando" $(r=-, 185, p<0.05)$.

Tabla 2

Coeficiente de correlación entre empatía y creencias irracionales.

\begin{tabular}{lcc}
\multicolumn{1}{c}{ Creencias irracionales } & \multicolumn{2}{c}{ Empatía } \\
\cline { 2 - 3 } Afecto y aprobación & $-0,122$ & 0,170 \\
Competencia absoluta &,$- 187^{*}$ & 0,035 \\
Relatividad de los actos & $-0,115$ & 0,198 \\
Expectativas sociales & 0,072 & 0,424 \\
Control externo & $-0,017$ & 0,846 \\
Continuidad de efectos &,$- 185^{*}$ & 0,038 \\
\hline
\end{tabular}

La correlación es significativa al nivel 0.01 


\section{DISCUSIÓN}

El incremento de los delitos sexuales así como el daño físico y psicológico causado a las víctimas es una preocupación social, para las instituciones implicadas y también para los investigadores. Tagle y Juárez (2014) consideran que los agresores sexuales presentan características significativas que llevan a cometer el delito: una inadecuada empatía, distorsiones cognitivas, falta de control de impulsos y el uso de mecanismos para justificar acciones. Además presentan un déficit en la capacidad para entender y comprender el sufrimiento de la víctima, debido a la percepción errónea respecto a esta en determinadas situaciones, dando lugar a cometer los delitos sexuales sin presencia de culpa. En este sentido el presente estudio plantea la vinculación de la empatía con las creencias irracionales.

Respecto a la relación entre empatía y la creencia de afecto y aprobación; el coeficiente de Spearman muestra un grado de intensidad de $r=-0,122(p>0,05)$ que indica que no existe relación significativa. Evidentemente, en este grupo de personas, la empatía, no se asocia a la necesidad de ser querido y aprobado por todas las personas significativas para uno. Esbec (2000) menciona que muchos de los agresores presentan un trastorno ansioso - evitativo, fracasando en sus relaciones interpersonales, anticipándose al fracaso y mostrándose deprimidos; así mismo menciona que no desarrollan suficientes habilidades sociales y presentan autoestima muy pobre. En el estudio realizado por Urquiza y Casullo (2005), se obtiene como resultado una correlación negativa entre la presencia de sentimientos de compasión y preocupación empática y la tendencia a razonar de manera prosocial, esperando la aprobación de otros; es decir que el hecho de que ellos entienden cognitivamente la necesidad de aprobación y les gustaría ser aprobados por los demás, no garantiza que sus conductas sean las más adecuadas en su entorno social.

Por otro lado, se encontró relación débil, negativa y significativa entre la creencia de competencia absoluta y la empatía $\left(r=-, 187^{*} ; p<0.05\right)$. Esto indica que a mayor presencia de pensamientos respecto a "debemos ser absolutamente competentes, inteligentes y eficaces en todos los aspectos posibles", el nivel de empatía disminuirá. Evidentemente la búsqueda de la competitividad con los demás afecta en el nivel de empatía en los internos por delito contra la libertad sexual; esto debido a que, mientras más sea su deseo por alcanzar la superioridad, más se centrarán en sus propios ideales y beneficios de forma egoísta, dejando de lado a los demás sin considerar las debilidades o sufrimiento de los otros, estableciendo relaciones negativas, vacías y destructivas, utilizándolos sin remordimiento para alcanzar su objetivo. En el estudio realizado por Samper, Calatrava y Martí (1998) a una población estudiantil, se encuentra que existe una tendencia a que, mientras mayor sea el grado de instrucción, mayor será la capacidad para comprender la situación de la víctima y ponerse en su lugar. Sin embargo; Villanueva, Moreno, Orozco, Soto y Vargas (2008) mencionan que la constante necesidad de sobresalir en el grupo a través de la exigencia propia, hace que las personas recluidas en cárceles pierdan la noción de la realidad y se exijan demasiado en determinados actos, eventos o situaciones dentro de los centros penitenciarios, para lograr sentirse libres a pesar del encierro y mostrarse sobresaliente en un grupo considerado poco exigente; causando así que no tomen en cuenta los sentimientos de los demás para conseguir lo que se proponen.

La empatía no se relacionó significativamente con la creencia de relatividad de los actos ( $r=-$ $0,115 ; p>0.05)$, la creencia de expectativas sociales (rho $=0,072 ; p>0.05)$ y la creencia de control externo $(r=-0,017 ; p>0.05)$. Esto representa que la empatía en los internos por delito contra la libertad sexual evaluados, fue independiente de dichas creencias irracionales. Este resultado podría deberse a la cantidad de participantes, ya que al ser una población con rasgos psicopatológicos, esta cantidad no nos permite tener resultados claros y significativos. Por otro lado Bejarano (2016) menciona que la permanencia por determinado tiempo en prisión, causa en los internos desadaptación social, la cual hace que se trastoque la noción de "seres sociales", presentando así dificultades para readaptarse y vivir en comunidad, aspecto que podría haber generado alteraciones en las actitudes al momento de responder los cuestionarios. Además de ello, este resultado se puede explicar debido a ciertas características personales de la muestra. En este sentido Esbec (2000) afirma que los esquemas cognitivos de los agresores sexuales, minimizan su responsabilidad y justifican su conducta, atribuyendo la culpa a factores externos y a la propia víctima. Así mismo; en el Manual 
Diagnostico y Estadístico de los Trastornos Mentales (Frances, 1995) se menciona que por lo general a este tipo de agresores no les interesa como sean las personas que los rodean mientras les ayude a cumplir sus objetivos. Otro factor importante es que debido a la falta de homogeneidad en la muestra, ya que cada uno presenta su propia personalidad y circunstancias biográficas, impidiendo así poder distinguir realmente sus conductas, se muestran de forma impredecible. Así mismo los rasgos psicopatológicos más frecuentes mencionan que este tipo de agresores presenta un alto índice de egocentrismo y manipulación, siendo encantadores y ocultando su verdadero yo ante los demás (Esbec, 2000). De igual forma Larrota y Rangel (2013) mencionan que los agresores sexuales presentan dificultad en las relaciones interpersonales y que para conseguir alguna cercanía con su entorno utiliza el engaño y la manipulación con los demás, creando un ambiente propicio para ellos en donde no puedan ser sorprendidos, utilizando su capacidad de planeación y encubrimiento para generar confianza en los demás. En este sentido Garrido (2006) menciona que una persona con rasgos de psicopatía es caracterizada por la manipulación, ya que esta al ser relacionada con su inteligencia y astucia, consigue la manipulación de otras personas. Una característica significativa de este tipo de personas es que lo lógico para ellos es la consecución de sus fines; saben diferenciar entre el bien o el mal, conocen sobre normas y leyes, pero no les brindan importancia alguna. Del mismo modo no muestra importancia hacia el dolor o el sufrimiento que sus acciones generan en los demás, lo único relevante para estas personas es lo que van a sacar de sus acciones. Así mismo se menciona que los agresores sexuales poseen un estilo de vida que puede ser considerado como una máscara social, ya que utilizan la manipulación mostrándose como personas ocupadas, productivas, versátiles, optimistas y espontáneas; en el ámbito familiar son juguetones, con convicciones altas, llenas de gratitud, por otro lado también presentan problemas en el manejo de la superficialidad e impulsividad. Esta mascara social permite que los agresores sexuales generen la intimidación en su víctima y en las demás personas de su entorno (Colectivo Arción, 2014)

Finalmente, sí se encontró relación débil, negativa y significativa entre la creencia de continuidad de efectos y empatía ( $r=-, 185^{*}$; $\mathrm{p}<0.05)$. Evidentemente, a mayor presencia de pensamientos respecto a "si algo afectó fuertemente en alguna ocasión nuestra vida, siempre la seguirá afectando" en los recluidos, menor fue el nivel de empatía. Probablemente, el hecho de haber sido víctimas de traumas en su infancia y adolescencia, genera en ellos sentimientos de resentimiento y odio hacia los demás; los cuales hacen que, cuando observan en otras personas situaciones dolorosas y similares a las de su pasado no pueden mostrar una adecuada empatía hacia ellos, ya que parte de sus patrones cognitivos hacen que crean que los demás también deben sufrir.

En este sentido Lescano (2005) menciona que los hechos pasados que generan malestar psicológico en el presente contienen síntomas constrictivos o evitativos, tales como la insensibilidad al dolor, incapacidad de respuesta, desapego o despersonalización, mostrando en el presente aturdimiento de la receptividad emocional, lo cual afecta sus relaciones interpersonales. Por ejemplo: estas personas miran con resentimiento su pasado, deseando haber vivido experiencias distintas que les permitan ser como ellos realmente quisieron ser. Así mismo Larrota y Rangel (2013) menciona que los agresores sexuales provienen de hogares disfuncionales, en los cuales han sido víctimas de diversas circunstancias tales como el maltrato físico por parte de los padres, problemas de alcoholismo e ingesta de drogas; de igual modo se menciona que dentro del ámbito sexual, estas personas han sido expuestas a material pornográfico para incrementar su interés sexual desde una corta edad, pensamientos machistas y degradantes hacia la mujer, conductas sexuales desviadas realizadas por sus progenitores las cuales han sido imitadas por ellos; finalmente Parrado (citado por Pereyra, 2017), en un estudio realizado con agresores sexuales, menciona el $100 \%$ de estos fueron violentados sexualmente, y a pesar de ser una situación que les genera dolor; su mecanismo de afrontamiento frente a esta situación es la actividad sexual violenta.

En conclusión; los resultados encontrados en la investigación evidencian que existe relación significativa negativa entre la empatía y las creencias irracionales de competencia absoluta y continuidad de efectos. Por otro lado, se encontró que no existe relación significativa entre la empatía y las creencias irracionales de afecto y aprobación, relatividad de los actos, expectativas sociales y control externo. En este 
sentido Gery, Miljkovitich, Berthoz y Soussignan (2009) mencionan que los agresores sexuales tienden a presentar niveles menores de empatía en relación a otros delincuentes con diferentes delitos.

\section{Declaración de financiamiento y de conflictos de interés:}

El estudio fue financiado por los autores, quienes declaran no tener conflictos de interés.

\section{Correspondencia}

Lisseth Brigitte Casariego More

Correo electrónico: lissethcasariego@upeu.edu. pe

Rudy Mijael Jara Cueto

Correo electrónico: rudyjara@upeu.edu.pe

\section{REFERENCIAS BIBLIOGRÁFICAS}

Bejarano, G. (2016). Sanción penal, efectos carcelarios y reincidencia en delitos sexuales. Revista de Psicologia, 16, 87-101. Recuperado de http://www.scielo.org.bo/pdf/rip/n16/n16_a06. pdf

Cepeda, Z. Y. (2012). Empatía y distorsiones cognitivas en personas no reclusas y en condenados por delitos sexuales y delitos violentos en Boyacá. (Tesis de maestría inédita). Universidad Nacional de Colombia, Bogotá.

Colectivo Arción (2014). Delito sexual "estupro". Visión criminológica- criminalística, 1, 18-25.

Davis, M. H. (1996). Empathy:A social psychological approach. Boulder, CO: Westview Press.

Ellis, A. y Harper, R. (1975). A New Guide to Rational Living. Hollywood: Wilshire Book Company.

Esbec, E. (2000). Evaluación psicológica de la víctima. En E. Esbec y G. Gómez-Jarabp.Madrid: Edisofer.

Fernández, Y., Marshall, W., Lightbody, S. y O’Sullivan, C. (1999). The Child molester Empathy Measure. Sexual Abuse. A Journal of Research and Treatment, 11, 17-31.Frances, A. (1995). DSM-IV audio review. Washington, DC: APPI.

Garrido, V. (2006). El Psicópata. Un camaleón en la sociedad actual. Madrid: Editorial Algar.
Gery, I., Miljkovitich, R., Berthoz, S. \& Soussignan, R. (2009). Empathy and recognition of facial expressions of emotion in sex off enders, nonsex off enders and normal controls. Psychatry Research, 165, 252-262.

Hernández, R., Fernández, C. y Baptista, P. (2006). Metodología de la Investigación. México: McGraw-Hill.

Instituto Nacional de Estadística e Informática. (2015). Estadísticas sobre Violencia Familiar y Sexual, Violencia contra la Mujer y Feminicidio en el Perú (N¹26 /2014-2015). Recuperado de http://www2. congreso.gob.pe/sicr/cendocbib/con4_uibd. nsf/4D6FF68892487BCF05257E2E005F78D 3/\$FILE/INFTEM126-2014-2015.pdf

Larrota, R., y Rangel, K. (2013). El agresor sexual. Aproximación teórica a su caracterización. Informes Psicológicos, 13 (2), 103-120.

Lescano, R. (2005). Trauma y EMDR: Un Nuevo Abordaje Terapéutico. Buenos Aires: Emdria Latinoamericana.

Marshall, W. (2004). Adult sexual ofender against women. In C. R. Hollin (ed.), The essential Handbook of ofender Assessment and Treatment. Chichester: Wiley.

Organización de las Naciones Unidas. (2015). Violencia contra las mujeres. Recuperado de http://www.unwomen.org/es/digital-library/ multimedia/2015/11/infographic-violence-againstwomen.

Organización Mundial de la Salud. (2015). Violencia de pareja y violencia sexual contra la mujer. Recuperado de http://www.who.int/mediacentre/ factsheets/fs239/es/

Ortiz, M., Sánchez, L. y Cardenal, V. (2002). Perfil psicológico de delincuentes sexuales. Un estudio clínico con el MCMI-II de Th. Millon. Revista de psiquiatría, facultad de medicina de Barcelona, 29(3), 144-153. Recuperado de: http://www.uma. es/psicologia/docs/eudemon/investigacion/perfil_ psicologico_de_delin cuentes_sexuales.pdf

Pereyra, G. (2017). Un estudio con abusadores: el $100 \%$ fueron niños abusados. El observador. Recuperado de https://www.elobservador.com. uy/un-estudio-abusadores-el-100-fueron-ninosabusados-n1058253

Samper, P., Calatrava, I. y Martí, M. (1998). Razonamiento Moral y Empatía. I Jornadas de Psicología del Pensamiento, 1, 389-404.

Tagle, E., Juarez, E. (2014). Criminología sexual. Revista del Instituto de Ciencia Jurídicas de Puebla, México, 34(1), 141-165. 
Efectividad del programa educativo "Mi amigo y yo, unidos contra la TB" en la prevención de tuberculosis a estudiantes de una institución educativa pública, Ate- Vitarte, Lima

Urquiza, V. y Casullo, M. (2005). Empatía, Razonamiento Moral y Conducta Prosocial en Adolescentes. Anuario de Investigación, 8, 297302. Recuperado de http://www.scielo.org.ar/pdf/ anuinv/v13/v13a31.pdf

Villanueva, I., Moreno, A. Orozco, L. Soto, L. y Vargas, Y. (2008). Creencias irracionales de condenados por delitos sexuales recluidos en la cárcel el Bosque de Barranquilla. Revista Psicogente, 9(15), 95-105. Recuperado de http://132.248.9.34/hevila/Psicogente/2006/vol9/ no15/6.pdf.

Recibido: 05/03/18

Aceptado: 11/09/18 